\title{
Open science requires an open internet
}

\author{
Niall W Duncan \\ Brain and Consciousness Research Centre, Taipei Medical University, Taiwan \\ niall.w.duncan@gmail.com
}

The movement towards open scientific practices can be said to have two main motivations: improving the quality of scientific work and making that work more democratic. These motivations compliment each other as making scientific research more available means that there are people with wider ranges of expertise and experience responding to it, leading to insights that would not otherwise been had. There is also a moral motivation for making science open to all. This may be based pragmatically on the fact that the public fund the majority of research and so deserve access to it, or on a more idealistic idea that science is a public good benefiting humanity as a whole.

Underlying both aims are efforts to change the way that scientific information is made available and discussed. Instead of placing knowledge behind barriers controlled by for-profit publishers, open scientific practices aim to make it freely available to all. This is being done through a combination of open-access publishing and the posting of papers to pre-print servers. At the same time, the movement aims to alter in different ways the ways that work is appraised, complementing the traditional peer-review process with community review both before and after publication. Finally, there are attempts to widen access to conferences through virtual contributions, allowing those without the means or opportunity to attend in person to benefit from and contribute to such discussions.

The common theme to all of these undertakings is that they rely upon online resources to achieve their aims. If these resources are not available to everybody then it could be said that open science becomes less open ${ }^{1}$. Making publications freely available and accessible requires that they be hosted somewhere that everyone has access to and which can be found. If people find the hosting service blocked or invisible to their search engines then the papers remain as inaccessible as ever. Similarly, the widening of voices able to discuss and contribute to science is only as wide as the number of people who are able to view the blogs or social media services that the discussion is taking place on. Without access to these, people remain unable to contribute to non-traditional peerreview and unable to add their voice to virtual discussions or conferences.

1 Of course, almost half of the world's population is estimated to have no access to the internet at all (https://www.internetworldstats.com/stats.htm). This is changing but should be borne in mind. 
For most of those with internet access, connecting to a full range of hosting and social media services is not currently a problem. This is not the case everywhere, however, and so it must be remembered that the openness of the internet that many are accustomed to can be removed. We tend to associate such actions with authoritarian regimes but they can occur in places that would be assumed to be more liberal. In the United Kingdom, for example, websites can currently be restricted based on the content that they contain ${ }^{2}$. Where blogs or social media discussions of particular areas of research fall into the filtered categories there is scope for them too being blocked, if only accidentally. As well as such government action, commercial considerations may also come into play in limiting access to open science. Where internet providers are given scope to modify access conditions for their users there is the risk that they choose to prevent access to particular hosting companies with whom they may be in competition or seek to gain an advantage over. Such decisions could have wide-ranging effects as the large hosting companies frequently provide backend services for seemingly unrelated websites.

Given that unrestricted internet access is afforded at the behest of governments and internet companies, those that seek to follow or promote open science practices should aim to be aware of policy as it stands in different countries and any proposals to change this. This will allow an appreciation of current limits to open science that deny some of its advantages to sections of the world and prevent voices from those regions contributing at this time. It will also allow them to be aware of proposals that threaten the way of doing science that they wish to adopt and potentially organise to argue against any such changes. Together these steps can help secure and extend the democratisation and improvement of science that the open science movement aims for.

2 Types of content that are currently restricted include items relating to sex, eating disorders, and suicide. 